\title{
Using the trans-lamina terminalis route via a pterional approach to resect a retrochiasmatic craniopharyngioma involving the third ventricle
}

\author{
Alexander G. Weil, MD, FRCSC, Thomas Robert, MD, Sultan Alsaiari, MD, Sami Obaid, MD, \\ and Michel W. Bojanowski, MD FRCSC
}

Division of Neurosurgery, Department of Surgery, Notre Dame Hospital, Montreal, QC, Canada

\begin{abstract}
Retrochiasmatic craniopharyngiomas involving the anterior third ventricle are challenging to access. Although the pterional approach is a common route for suprasellar lesions, when the craniopharyngioma extends behind the chiasma into the third ventricle, access is even more difficult, and the lamina terminalis may offer a good working window. The translamina terminalis approach provides direct access to the retrochiasmatic portion of the tumor with minimal brain retraction and no manipulation of the visual nerves. In this video, we emphasize the utility of using the lamina terminalis corridor to resect the retrochiasmatic intraventricular portion of a craniopharyngioma.
\end{abstract}

The video can be found here: https://youtu.be/hrLNCOhDKe4.

KEYWORDS craniopharyngioma; retrochiasmatic; third ventricle; pterional; translamina terminalis; video 